\title{
INFINITE RADICALS IN THE COMPLEX PLANE ${ }^{1}$
}

\author{
GEORGELLEN SCHUSKE AND W. J. THRON
}

1. Introduction. If $\left\{a_{n}\right\}$ is a sequence of complex numbers, the sequence $\left\{u_{n}\right\}$, where

$$
u_{n}=\left(a_{1}+\left(a_{2}+\cdots+\left(a_{n}\right)^{1 / 2}\right)^{1 / 2}\right)^{1 / 2}
$$

is called an infinite radical. The numbers $a_{j}$ are the elements of the infinite radical, and the $u_{j}$ are called partial radicals.

Throughout this paper $a^{1 / 2}$ will mean that square root whose real part is positive or zero. If $a$ is a negative real number, $a^{1 / 2}$ will be defined to be on the positive imaginary axis. A set which is open and connected is called an open region, and an open region with part or all of its boundary is called a region. A region $A$ is called a convergence region for an infinite radical $\left\{u_{n}\right\}$ if the assumption that all the elements $a_{j}$ lie in $A$ is sufficient for the convergence of $\left\{u_{n}\right\}$. A region $A$ is called a conditional convergence region if the assumption that all the elements $a_{j}$ lie in $A$, together with another condition which restricts the rapidity of growth of $\left|a_{j}\right|$, insures the convergence of $\left\{u_{n}\right\}$.

It is not difficult to prove that a necessary and sufficient condition for a sequence $\left\{s_{n}\right\}$ of real numbers to be an infinite radical $\left\{u_{n}\right\}$ is that $\left\{s_{n}\right\}$ be a monotonically nondecreasing sequence of non-negative numbers. However, if $\left\{s_{n}\right\}$ is a sequence of complex numbers, the situation is not so simple. If $P_{n}$ is the region from which $s_{n}$ may be chosen, $P_{n} \subset P_{n-1}$, for every $n$, but each $P_{n}$ depends on the entire set of numbers $s_{1}, s_{2}, \cdots, s_{n-1}$, and therefore necessary and sufficient conditions are rather involved and not very meaningful geometrically.

Vieta was probably the first to conceive of the idea of an infinite radical $[1$, p. 595]. The famous infinite product

$$
\begin{aligned}
2 / \pi= & 1 / 2^{1 / 2} \cdot\left(1 / 2+1 / 2(1 / 2)^{1 / 2}\right)^{1 / 2} \\
& \cdot\left(1 / 2+1 / 2\left(1 / 2+1 / 2(1 / 2)^{1 / 2}\right)^{1 / 2}\right)^{1 / 2} \ldots
\end{aligned}
$$

appeared in 1593 in his derivation for the area of a unit circle. The unconditional convergence of this product was proved by Rudio in 1890 [7].

Presented to the Society, November 19, 1960; received by the editors September 2, 1960.

1 This research was supported by the United States Air Force under contract no. AF 49(638)-100 monitored by the AF Office of Scientific Research of the Air Research and Development Command. 
Ramanujan [6], in 1911, obtained values for certain specific infinite radicals, and Pólya [5], in 1916, gave a convergence criterion for infinite radicals with positive real elements. In 1935, Herschfeld [2] proved the following necessary and sufficient condition for the convergence of an infinite radical with positive real elements:

The sequence $\left\{u_{n}\right\}$ defined by

$$
u_{n}=\left(a_{1}+\left(a_{2}+\cdots+a_{n}^{1 / 2}\right)^{1 / 2}\right)^{1 / 2}, \quad a_{j}>0,
$$

converges if and only if there exists a finite upper limit

$$
\limsup _{n \rightarrow \infty} a_{n}^{2^{-n}}<\infty \text {. }
$$

The purpose of this paper is to determine conditional convergence regions for sequences $\left\{u_{n}\right\}$ with complex elements. The StieltjesVitali Theorem for normal families of functions is used, together with Herschfeld's criterion for infinite radicals with positive real elements. This method is similar to that used previously $[3 ; 4]$ in the case of continued fractions.

2. Element regions and value regions. If all the elements $a_{n}$ of the infinite radical $\left\{u_{n}\right\}$ lie in a region $A$ of the complex plane, $A$ is called an element region of the infinite radical. If the values of all partial radicals $u_{n}$ whose elements are in the region $A$ lie in a region $U$, this region $U$ is called a value region corresponding to the element region $A$. It should be noted that a value region may contain other points besides the values taken on by the partial radicals $u_{n}$.

Introduce the following iterative device for constructing an infinite radical $\left\{u_{n}\right\}$ :

$$
\begin{aligned}
T_{n}(z) & =T_{n-1}\left(t_{n}(z)\right), & & n>1, \\
T_{1}(z) & =t_{1}(z), & & \\
t_{n}(z) & =\left(a_{n}+z\right)^{1 / 2}, & & \text { for all } n .
\end{aligned}
$$

One readily sees that $T_{n}(0)=u_{n}$.

Let $\psi(z)$ be any function of $z$ and let $V$ be any set in the complex plane. Then $\psi(V)$ is understood to be the set of all points $\psi(z)$ where $z \in V$.

Lemma 2.1. If two regions $A$ and $U$ in the complex plane are given such that

$$
0 \in U,
$$

and 


$$
t_{n}(U) \subset U \text { if } a_{n} \in A,
$$

then $U$ is a value region corresponding to the element region $A$.

Proof. We shall use induction to prove that every partial radical $T_{n}(0)$ is in $U$ for any choice of the $a_{1}, a_{2}, \cdots, a_{n} \in A$.

By (i) and (ii), for any choice of $a_{1} \in A$,

$$
T_{1}(0) \in U \text {. }
$$

Assume that, for any choice of $a_{1}, a_{2}, \cdots, a_{N-1} \in A$,

$$
T_{N-1}(U) \subset U \text {. }
$$

By (ii), for any $a_{N} \in A$,

$$
t_{N}(U) \subset U
$$

Then

$$
T_{N}(U)=T_{N-1}\left(t_{N}(U)\right) \subset U
$$

for any choice of $a_{1}, a_{2}, \cdots, a_{N} \in A$. Therefore, for every $n, T_{n}(z) \in U$ for any choice of elements from $A$ and any $z \in U$. Since $0 \in U$, it follows that $T_{n}(0) \in U$ for every $n$ and any choice of elements from $A$.

THEOREM 2.1. Let $\theta$ be an arbitrary fixed number, $0<\theta<\pi$. Let $a \in A$ if and only if

$$
|a|>0 \text { and }-\theta<\arg a<g(\theta),
$$

where

$$
g(\theta)=\left\{\begin{array}{lll}
\pi-\theta / 2 & \text { when } & 0<\theta \leqq 2 \pi / 3 \\
2(\pi-\theta) & \text { when } & 2 \pi / 3 \leqq \theta<\pi
\end{array}\right.
$$

Let $z \in U$ if and only if

$$
|z| \geqq 0 \text { and }-\theta / 2<\arg z<g(\theta) / 2 .
$$

Then $U$ is a value region corresponding to the element region $A$.

Proof. Fix $a_{n} \in A$. Then $a_{n}+U$ is an angular opening contained in $A$, and

$$
\left(a_{n}+U\right)^{1 / 2} \subset U \text {. }
$$

An application of Lemma 2.1 completes the proof of the theorem.

3. The principal theorem. Define sequences $\left\{f_{n}(z)\right\}$ and $\left\{\phi_{n}(z)\right\}$ as follows: 


$$
\begin{aligned}
f_{n}(z) & =\left|a_{n}\right| e^{i z \alpha_{n}} \\
\phi_{n}(z) & =\left(f_{1}(z)+\left(f_{2}(z)+\cdots+\left(f_{n}(z)\right)^{1 / 2}\right)^{1 / 2}\right)^{1 / 2}
\end{aligned}
$$

where

$$
a_{n}=\left|a_{n}\right| e^{i a_{n}}, \text { and }-\pi<\alpha_{n} \leqq \pi .
$$

These sequences will be used in the proof of the principal theorem, which follows.

TheOREM 3.1. Let $\theta$ be a fixed number, $0<\theta<\pi$. Let $\epsilon$ be an arbitrarily small fixed positive number, $0<\epsilon<\min [\theta, g(\theta)]$. Let $a \in A_{\epsilon}$ if and only if

$$
|a|>0 \text { and }-\theta+\epsilon<\arg a<g(\theta)-\epsilon,
$$

where $g(\theta)$ is defined as in Theorem 2.1. Then the infinite radical $\left\{u_{n}\right\}$, where

$$
u_{n}=\left(a_{1}+\left(a_{2}+\cdots+a_{n}^{1 / 2}\right)^{1 / 2}\right)^{1 / 2},
$$

converges if

$$
a_{n} \in A_{\epsilon}
$$

and

$$
\limsup _{n \rightarrow \infty}\left|a_{n}\right|^{2^{-n}}<\infty .
$$

Proof. Define the region $R$ as follows: $z=x+i y \in R$ if and only if

$$
\begin{gathered}
-\delta<x<1+\delta, \text { where } \delta=\epsilon /(\pi-\epsilon), \\
|y|<1 .
\end{gathered}
$$

Let $a_{n} \in A_{\text {e. }}$ Assume first that

$$
0 \leqq x<1+\delta .
$$

Then, since

$$
\arg f_{n}(z)=x \arg a_{n},
$$

we have

$$
-\theta<(1+\delta)(-\theta+\epsilon)<\arg f_{n}(z)<(1+\delta)[g(\theta)-\epsilon]<g(\theta) .
$$

Next, assume that $-\delta<x<0$. Then, since

$$
\frac{\epsilon}{\pi-\epsilon}<\min \left[\frac{\theta}{g(\theta)-\epsilon}, \frac{g(\theta)}{\theta-\epsilon}\right]
$$


it follows that

$$
-\theta<-\delta[g(\theta)-\epsilon]<\arg f_{n}(z)<-\delta(-\theta+\epsilon)<g(\theta) .
$$

Thus, if $z \in R$ and $a_{n} \in A_{\epsilon}, f_{n}(z)$ lies in the region $A$ of Theorem 2.1, since $f_{n}(z)$ clearly satisfies the requirement $\left|f_{n}(z)\right|>0$. Further,

$$
\left(f_{n}(z)+U\right)^{1 / 2} \subset U,
$$

where $U$ is the region $U$ of that theorem. Then $U$ is a value region for $\left\{\phi_{n}(z)\right\}$. Hence, the expression

$$
f_{m}(z)+\left(f_{m+1}(z)+\cdots+\left(f_{q}(z)\right)^{1 / 2}\right)^{1 / 2}, \quad m \leqq q,
$$

cannot be zero or a negative real number for any $m, q$.

In general, if $h(z)$ is any holomorphic function of $z$ in an open region $S$, the function $(b+h(z))^{1 / 2}$ has a branch point at any value of $z$ for which $h(z)=-b$. If $S$ is such that for $z \in S, b+h(z)$ is not zero or a negative real number, then $(b+h(z))^{1 / 2}$ is holomorphic.

Clearly $f_{n}(z)$ is holomorphic for $z \in R$. By the preceding remarks, $\phi_{n}(z)$ is holomorphic for $z \in R$. According to a theorem due to Montel (see, for example, [8, p. 218]), a family of holomorphic functions is normal in a region $S$ if there exist two distinct values which are not assumed by any member of the family for $z \in S$. Since $\phi_{n}(z) \in U$ for every $n$ and for all $z \in R,\left\{\phi_{n}(z)\right\}$ is a normal family in $R$.

Denote by $\Delta$ the set of points $z \in R$ for which $x=0$. Then, if $z \in \Delta$, $f_{n}(z)$ is real and positive, and

$$
f_{n}(z)=\left|a_{n}\right| e^{-y \alpha_{n}}, \quad z \in \Delta .
$$

It is at this point that Herschfeld's criterion can be used. Thus, for $z \in \Delta$, the sequence $\left\{\phi_{n}(z)\right\}$ converges if

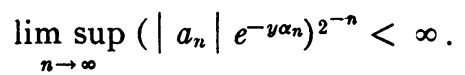

Therefore, condition (ii) insures the convergence of $\left\{\phi_{n}(z)\right\}$ for $z \in \Delta$.

We have proved that $\left\{\phi_{n}(z)\right\}$ is a normal family in $R$, and that there is an infinite set of points $\Delta$ having a limit point in $R$, such that, if $z \in \Delta, \lim _{n \rightarrow \infty} \phi_{n}(z)$ exists. Then by the Stieltjes-Vitali Theorem (see, for example, $\left[8\right.$, p. 142]), $\left\{\phi_{n}(z)\right\}$ converges uniformly in every compact set $K \subset R$, and in particular $\left\{\phi_{n}(1)\right\}$ converges. Since $\left\{\phi_{n}(1)\right\}$ is the original sequence $\left\{u_{n}\right\}$, the proof of the theorem is complete.

4. Best regions. It is possible that the conditional convergence regions $A_{\epsilon}$ obtained in Theorem 3.1 do not have the widest possible angular openings. We shall not attempt to answer this question in 
general, but we shall show by examples that for $\theta=2 \pi / 3$ there are infinite radicals which satisfy condition (ii) of Theorem 3.1 and yet diverge if the angular opening of the element region is increased beyond that of $A_{\epsilon}$. In this sense, $A_{\epsilon}$ is a best region. Here, $a \in A_{\boldsymbol{\epsilon}}$ if and only if, for arbitrarily small $\epsilon, 0<\epsilon<2 \pi / 3$,

$$
|a|>0 \text { and }|\arg a|<2 \pi / 3-\epsilon .
$$

For our first example, let the elements of $\left\{u_{n}\right\}$ be

$$
a_{2 n-1}=e^{i 2 \pi / 3}, \quad a_{2 n}=e^{-i 2 \pi / 3} .
$$

Then $\left\{u_{n}\right\}$ diverges, since, for every $n$,

$$
u_{2 n}=0 \text { and } u_{2 n-1}=e^{i \pi / 3} .
$$

Our next example shows that the $\epsilon$ in the definition of $A_{\epsilon}$ cannot be omitted and that the "buffer space" to the right of the rays $\gamma= \pm 2 \pi / 3$ is really necessary. Let the elements of $\left\{u_{n}\right\}$ be

$$
\begin{aligned}
a_{2 n-1} & =\exp \left\{i\left[2 \pi / 3-(\eta / 2)^{2^{n}}\right]\right\}, \\
a_{2 n} & =\exp \left\{-i\left[2 \pi / 3-(\eta / 2)^{2^{n}}\right]\right\},
\end{aligned}
$$

where $\eta$ is an arbitrary real number such that $0<\eta<1 / 4$. It can be proved that, for every $n$,

$$
\left|u_{2 n}\right|<\eta \text { and }\left|u_{2 n-1}-e^{i \pi / 3}\right|<\eta .
$$

Hence, $\left\{u_{n}\right\}$ does not converge.

\section{BIBLIOGRAPHY}

1. Moritz Cantor, Vorlesungen über Geschichte der Mathematik, vol. 2, 2d ed., Leipzig, 1900.

2. Aaron Herschfeld, On infinite radicals, Amer. Math. Monthly vol. 42 (1935) pp. 419-429.

3. Walter Leighton and W. J. Thron, Continued fractions with complex elements, Duke Math. J. vol. 9 (1942) pp. 763-772.

4. J. F. Paydon and H. S. Wall, The continued fraction as a seguence of linear transformations, Duke Math. J. vol. 9 (1942) pp. 360-372.

5. G. P6lya, Aufgabe 501, Arch. Math. Phys. vol. 24 (1916) p. 84.

6. Srinivasa Ramanujan, J. Indian Math. Soc. Problem 289, vol. 3 (1911) p. 90; Solution, vol. 4 (1912) p. 226.

7. F. Rudio, Über die Konvergenz einer von Vieta herrührenden eigentümlichen Produktentwickelung, Z. Math. Phys. vol. 36 (1890) pp. 139-140.

8. W. J. Thron, Introduction to the theory of functions of a complex variable, New York, John Wiley and Sons, Inc., 1953.

UNiversity of Colorado 